\title{
Incidence of Pinhole Type Durotomy and Subsequent Cerebrospinal Fluid Leakage Following Simple Laminectomy
}

\author{
Faizan Imran Bawany ${ }^{1}$, Muhammad Emaduddin ${ }^{2}$, Manzar Shahid ${ }^{1}$, Mehwish Hussain ${ }^{1}$, \\ Mohammad Yousuful Islam ${ }^{1}$, Muhammad Shahzeb Khan ${ }^{1}$ \\ ${ }^{1}$ Dow University of Health Sciences, Karachi, Pakistan \\ ${ }^{2}$ Neurosurgery Department, Civil Hospital, Karachi, Pakistan
}

\section{Study Design: Cross sectional study.}

Purpose: The purpose of this study was to determine the incidence and the associated risk factors of pinhole type of durotomy and cerebrospinal fluid (CSF) leakage following a simple laminectomy for spinal stenosis.

Overview of Literature: The incidence of spinal stenosis is expected to rise with increasing life expectancy. Moreover, lumbar spinal stenosis is the most common indication for spinal injury in the geriatric population. It is therefore important to identify and prevent the risks associated with laminectomy, the most widely used surgical procedure for spinal stenosis. The serious complication of incidental dural tear or durotomy and subsequent CSF leakage has not been studied in the region of Southeast Asia.

Methods: In this cross sectional study, we included 138 adult patients (age $>18$ years), who underwent a simple laminectomy for lumbar stenosis between 2011 and 2012. CSF leakage was the main outcome variable. Patients' wounds were examined for CSF leakage up to 1 week postoperatively.

Results: The incidence of pinhole type durotomy and subsequent CSF leakage in our region was $8.7 \%$. Univariate analysis showed that hypertension, diabetes and smoking were significantly associated with durotomy and increased CSF leakage by 16.72, 44.25, and 33.71 times, respectively. Multivariate analysis showed that only smoking and diabetes significantly increased the chances of leakage. Conclusions: Glycemic control and cessation of smoking prior to a simple laminectomy procedure reduced the incidence of a dural tear. Larger clinical studies on this lethal complication are required.

Keywords: Cerebrospinal fluid leak; Dura mater; Incidence; Laminectomy; Risk factors

\section{Introduction}

Lumbar stenosis denotes the narrowing of the spinal canal. It occurs due to degenerative changes characterized by ligamentum flavum (ligaments of the spine) hypertrophy, bulging of the intervertebral disc and osteophytic disease of the vertebral body, eventually leading to compression of the neural elements [1,2]. Patients may present with back pain, neurogenic or pseudoclaudication and radiculopathy [1-3]. Lumbar magnetic resonance imaging (MRI) is used to assess neural tissue compression, whereas computed tomography scan is used to evaluate spinal

Received Oct 26, 2014; Revised Dec 1, 2014; Accepted Dec 18, 2014

Corresponding author: Manzar Shahid

Dow University of Health Sciences, Baba-E-Urdu Road, Karachi 74200 - Pakistan

Tel: +92-3463288816, E-mail: manzar.shahid@hotmail.com 
canal diameter and assess the bone [1-3]. Initial treatment of lumbar stenosis is conservative $[1,2]$. Surgical decompression is undertaken when symptoms become severe despite medical management $[1,2,4]$. Laminectomy is the most commonly used procedure to decompress the neural elements that produce patients' symptoms [1,2]. Laminectomy is a procedure performed to surgically remove any compressive element responsible for the patients' symptoms, including the lamina or associated tissue.

The incidental dural tear or durotomy during laminectomy is a known complication that has severe postprocedural consequences. The incidence of durotomy ranges from $1 \%$ to $17 \%$ [5-7]. It commonly occurs due to a direct tear of the dura or excessive nerve root traction during removal of the extruded disc $[2,5,8]$. In the majority of cases of durotomy, the tear is detected during the initial surgical procedure and primary repair is compulsory. Occasionally, pinhole durotomies (inadvertent) may go unnoticed during the initial surgical procedure. This may result in cerebrospinal fluid (CSF) leakage after the surgery is completed, leading to symptoms such as postural headache, nausea and vomiting $[7,8]$. CSF leakage may also cause potential complications such as CSF fistula formation, pseudomeningocele, meningitis and arachnoiditis resulting in increased morbidity and prolonged hospital stay [5,7].

Lumbar spinal stenosis is the most common indication for spinal injury in the elderly and its incidence is on the rise with the increase in life expectancy [4]. Laminectomy, the most common surgical procedure has long term risks that can be identified and prevented. This study was the first of its kind in our region. We investigated the incidence and associated risk factors of pin-hole-type durotomy and CSF leakage after the initial surgical procedure.

\section{Materials and Methods}

The cross sectional study was conducted over a period of 2 years from 2011 to 2012 with the approval of the Institutional Review Board of Dow University of Health Sciences. We included 138 patients, adults (age $>18$ years), who underwent a simple laminectomy for lumbar stenosis. Patients that presented with symptoms of neurogenic claudication or radicular leg pain, with associated neurological signs which persisted for at least six weeks and were refractory to medical treatment, were selected for laminectomy. Neurogenic claudication was defined as a combination of low back pain and numbness that started or intensified on standing or walking and was relieved by sitting or lying down. An MRI was used to obtain evidence of compression of neural elements such as spinal nerves, thecal sac and the spinal cord.

Patients suffering from congenital neural tube or vertebral column defects, trauma and any spinal tumor were excluded from the study. Moreover, patients undergoing surgical procedures other than a laminectomy were also excluded. During a laminectomy, the posterior vertebral elements including the spinous process, interspinous ligaments along with the associated ligamentum flavum, lamina, disc and facet joints were removed. The main outcome variable was CSF leakage that was measured postoperatively. Patients' wounds were examined postoperatively for 1 week to observe soaking of wound dressings for any kind of leakage. A glucose dipstick test was performed to confirm the presence of CSF leakage. Informed consent was obtained from all competent patients and from the immediate attendants of incompetent patients. Patients' hospital records including demographic, operative and postoperative (presence of leakage) data were collected for analysis.

All descriptive measures were presented in terms of frequencies and percentages. A chi-square test of association was performed to assess the effects of the demographic and clinical characteristics on CSF leakage. Crude odds ratios (ORs) were computed to assess the likelihood of leakage due to significant factors. Factors that were significant at the $10 \%$ value of type I error in univariate analysis were inserted in a multivariate logistic regression model to obtain the adjusted odds ratio. SPSS ver. 21 (IBM Co., Armonk, NY, USA) was used to enter and analyze the data.

\section{Results}

A total of 138 patients were included in the study. Majority of patients were younger than 65 years of age $(n=134$, $97.1 \%)$, males ( $\mathrm{n}=86,62.3 \%)$, and overweight with a body mass index (BMI) of $25.0-29.9 \mathrm{~kg} / \mathrm{m}^{2}(\mathrm{n}=69,50 \%)$. The patients' characteristics were defined in Table 1.

According to our results, CSF leakage was presented in $8.7 \%(\mathrm{n}=12)$ of patients (Fig. 1). CSF leakage was significantly more in the $41-65$ years old age group $(n=8,66.7 \%$; $p=0.008)$ and in patients with a BMI of $30.0-34.9 \mathrm{~kg} / \mathrm{m}^{2}$ $(\mathrm{n}=7,58.3 \% ; p<0.001)$. Although insignificant, most of 
Table 1. Patients' characteristics

\begin{tabular}{|c|c|}
\hline Variable & No. $(\%)$ \\
\hline \multicolumn{2}{|l|}{ Age (yr) } \\
\hline $18-40$ & $66(47.8)$ \\
\hline $41-65$ & $68(49.3)$ \\
\hline$>65$ & $4(2.9)$ \\
\hline \multicolumn{2}{|l|}{ Sex } \\
\hline Male & $86(62.3)$ \\
\hline Female & $52(37.7)$ \\
\hline \multicolumn{2}{|l|}{ Level } \\
\hline L1-L2 & $1(0.7)$ \\
\hline $\mathrm{L} 2-\mathrm{L} 3$ & $8(5.8)$ \\
\hline L3-L4 & $9(6.5)$ \\
\hline L4-L5 & $93(67.4)$ \\
\hline L5-S1 & $27(19.6)$ \\
\hline \multicolumn{2}{|l|}{ Infection } \\
\hline No & $134(97.1)$ \\
\hline Yes & $4(2.9)$ \\
\hline \multicolumn{2}{|l|}{ Hematoma } \\
\hline No & $133(96.4)$ \\
\hline Yes & $5(3.6)$ \\
\hline \multicolumn{2}{|l|}{ Hypertension } \\
\hline No & $99(71.7)$ \\
\hline Yes & $39(28.3)$ \\
\hline \multicolumn{2}{|l|}{ Diabetes } \\
\hline No & $121(87.7)$ \\
\hline Yes & $17(12.3)$ \\
\hline \multicolumn{2}{|l|}{ Smoking } \\
\hline No & $96(69.6)$ \\
\hline Yes & $42(30.4)$ \\
\hline \multicolumn{2}{|l|}{ Drug use } \\
\hline No & $125(90.6)$ \\
\hline Yes & $13(9.4)$ \\
\hline \multicolumn{2}{|c|}{ Body mass index $\left(\mathrm{kg} / \mathrm{m}^{2}\right)$} \\
\hline$<18.5$ & 0 \\
\hline $18.5-24.9$ & $34(24.6)$ \\
\hline $25-29.9$ & $69(50)$ \\
\hline $30-34.9$ & $23(16.7)$ \\
\hline$\geq 35$ & $12(8.7)$ \\
\hline
\end{tabular}

the CSF leakage was at the level of L4-L5 ( $\mathrm{n}=10,83.3 \%)$. None of the patients developed an infection or a hematoma due to CSF leakage. Hypertension $(n=10,83.3 \%)$,

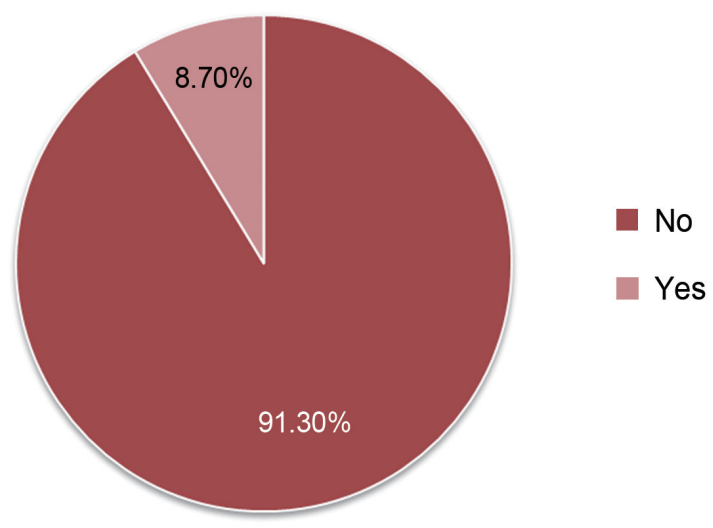

Fig. 1. Percentage distribution of cerebrospinal fluid leakage.

diabetes $(n=9,75 \%)$, and smoking $(n=11,91.7 \%)$ were significantly associated with CSF leakage $(p<0.001)$. However, no significant association was found between drug use ( $n=3,25 \%)$ and CSF leakage ( $p=0.087)$. These findings were shown in Table 2.

Odds ratio analyses were shown in Table 3. The crude analysis showed that the hypertensive patients had a 16.72 times higher chance of developing a CSF leakage than normotensive patients (OR, 16.72; 95\% confidence interval [CI], 3.47-80.70). Diabetic patients had 44.25 times higher likelihood of leakage than their normal counterparts (OR, 44.25; 95\% CI, 9.97-196.39). Smoking augmented the chances of leakage by 33.71 times (OR, 33.71; 95\% CI, 4.16-271.69). All these effects were statistically significant with $p<0.001$. Although insignificant $(p=0.087)$, drug use increased the chances of leakage by 3.87 times (OR, 3.87; 95\% CI, 0.90-16.61).

Hypertension and drug use were statistically insignificant even at $10 \%$ level of significance after adjusting for the effects of age and BMI. Nevertheless, smoking and diabetes were significantly associated with CSF leakage. The analysis revealed that smoking yielded a higher effect than diabetes on CSF leakage.

\section{Discussion}

Incidental durotomy and subsequent CSF leakage are adverse side effects of laminectomy and other lumbar decompressive surgeries. Incidence of durotomy has increased since the advent of spinal instrumentation along with the aggressive management of many spinal conditions [5]. The incidence of pinhole durotomy following a first-time laminectomy for spinal stenosis was $8.7 \%(n=12)$ 
Table 2. Comparison of percentage distribution of CSF leakage

\begin{tabular}{|c|c|c|c|}
\hline \multirow{2}{*}{ Variable } & \multicolumn{2}{|c|}{ CSF leakage } & \multirow{2}{*}{$p$-value } \\
\hline & No & Yes & \\
\hline Age (yr) & & & 0.008 \\
\hline $18-40$ & 64 (50.8) & $2(16.7)$ & \\
\hline $41-65$ & $60(47.6)$ & $8(66.7)$ & \\
\hline$>65$ & $2(1.6)$ & $2(16.7)$ & \\
\hline Sex & & & 0.503 \\
\hline Male & 78 (61.9) & $8(66.7)$ & \\
\hline Female & 48 (38.1) & 4 (33.3) & \\
\hline Spinal cord level & & & 0.547 \\
\hline $\mathrm{L} 1-\mathrm{L} 2$ & $1(0.8)$ & 0 & \\
\hline $\mathrm{L} 2-\mathrm{L} 3$ & $8(6.3)$ & 0 & \\
\hline L3-L4 & $9(7.1)$ & 0 & \\
\hline$\llcorner 4-\llcorner 5$ & 83 (65.9) & 10 (83.3) & \\
\hline L5-S1 & 25 (19.8) & $2(16.7)$ & \\
\hline Infection & & & $>0.999$ \\
\hline No & 122 (96.8) & $12(100)$ & \\
\hline Yes & $4(3.2)$ & 0 & \\
\hline Hematoma & & & $>0.999$ \\
\hline No & $121(96)$ & $12(100)$ & \\
\hline Yes & $5(4)$ & 0 & \\
\hline Hypertension & & & $<0.001$ \\
\hline No & $97(77)$ & 2 (16.7) & \\
\hline Yes & $29(23)$ & 10 (83.3) & \\
\hline Diabetes & & & $<0.001$ \\
\hline No & 118 (93.7) & $3(25)$ & \\
\hline Yes & $8(6.3)$ & $9(75)$ & \\
\hline Smoking & & & $<0.001$ \\
\hline No & $95(75.4)$ & $1(8.3)$ & \\
\hline Yes & 31 (24.6) & $11(91.7)$ & \\
\hline Drug use & & & 0.087 \\
\hline No & $116(92.1)$ & $9(75)$ & \\
\hline Yes & $10(7.9)$ & $3(25)$ & \\
\hline Body mass index $\left(\mathrm{kg} / \mathrm{m}^{2}\right)$ & & & $<0.001$ \\
\hline$<18.5$ & 0 & 0 & \\
\hline $18.5-24.9$ & $34(27)$ & 0 & \\
\hline $25-29.9$ & $66(52.4)$ & $3(25)$ & \\
\hline $30-34.9$ & $16(12.7)$ & $7(58.3)$ & \\
\hline$\geq 35$ & $10(7.9)$ & 2 (16.7) & \\
\hline
\end{tabular}

CSF, cerebrospinal fluid.

in our region. Guerin et al. [6] reported that 13 of 51 cases of durotomies were unrecognized during the initial surgi- cal procedure with the incidence of pinhole durotomy at $25 \%$. Our results showed that 41 to 65 -year-old patients 
Table 3. Effect of significant patient's characteristics

\begin{tabular}{|c|c|c|c|c|c|c|}
\hline \multirow{2}{*}{ Variable } & \multicolumn{3}{|c|}{ Crude OR } & \multicolumn{3}{|c|}{ Adjusted $\mathrm{OR}^{\mathrm{a})}$} \\
\hline & Times & $95 \% \mathrm{Cl}$ & $p$-value & Times & $95 \% \mathrm{Cl}$ & $p$-value \\
\hline Hypertension & 16.72 & $3.47-80.70$ & $<0.001$ & 4.40 & 0.18 & 108.87 \\
\hline Diabetes & 44.25 & 9.97-196.39 & $<0.001$ & 18.32 & 0.76 & 439.34 \\
\hline Smoking & 33.71 & 4.18-271.69 & $<0.001$ & 59.11 & 2.57 & $1,361.79$ \\
\hline Drug used & 3.87 & $0.9-16.61$ & 0.087 & 0.15 & 0.003 & 8.94 \\
\hline
\end{tabular}

$\mathrm{OR}$, odds ratio; $\mathrm{Cl}$, confidence interval.

${ }^{a)}$ Adjusted for age and body mass index.

were significantly more susceptible to durotomy and subsequent CSF leakage. However, Williams et al. [7] showed that durotomy was significantly more common in the elderly population (age, $>80$ years). In contrast to the findings of a previous study [8], our results showed that BMI has a significant impact on durotomy and subsequent CSF leakage.

Desai et al. [9] previously showed that gender, hypertension, diabetes and smoking have no significant association with durotomy. Our study demonstrated the same gender effect. However, our univariate analysis showed that hypertension, diabetes and smoking were significantly associated with durotomy and increased chances of CSF leakage by 16.72, 44.25, and 33.71 times, respectively. Furthermore, multivariate analysis indicated that only smoking (by 59.10 times, $p=0.011$ ) and diabetes (by 18.32 times, $p=0.073$ ) significantly increased the chances of leakage. Univariate analysis for the effect of diabetes on incidental durotomy in another study [8] also showed that diabetes is a significant risk factor for durotomy. However, diabetes had no significant association with durotomy on multivariate regression in the same study [8]. The multivariate analysis in our study indicated no significant effect of hypertension on CSF leakage following a laminectomy, in contrast to the earlier report that hypertension is a significant risk factor for dural tear following lumbar spinal decompression [10].

The link between smoking and delayed wound healing is well known [11]. Toxic substances present in cigarette smoke-particularly nicotine, carbon monoxide and hydrogen cyanide-may be responsible for delayed wound healing. Nicotine is a potent vasoconstrictor that decreases blood flow to the tissues resulting in ischemia and delayed wound healing. Carbon monoxide reduces oxygen delivery whereas cyanide inhibits oxidative phosphoryla- tion at the mitochondrial level. Adverse effects of diabetes on wound healing are also well reported in literature [12]. Increased blood glucose is associated with glycosylation of blood proteins and impaired functioning of immune cells necessary for wound healing. In short, both smoking and diabetes have a negative impact on wound healing and repair. A study by Ahn et al. [13] showed that smoking and diabetes are significant risk factors for dural tears, with a significant positive association between dural tear and smoking (OR, 3.4) and diabetes (OR, 2.3). According to the authors, smoking and diabetes decreased the viability and strength of fibrous connective tissues in abdominal fascia, which are similar to that of the dura. Therefore, cessation of smoking and adequate control of diabetes is encouraged prior to lumbar surgery to minimize the chances of a dural tear and subsequent CSF leakage.

Unfortunately, comparative randomized clinical trials indicating the benefits of one of several operative and nonoperative approaches for the treatment of pinhole durotomy and subsequent CSF leakage after the initial surgical procedure are lacking [5]. Some surgeons believe that immediate reoperation is the best approach once CSF leakage is observed while others believe that conservative management should be initiated first. Commonly used conservative approaches for CSF leakage management are spinal drainage and bed rest for 5-7 days. Another conservative approach is the blood patch that involves injection of $15 \mathrm{~mL}$ of autologous blood into the epidural space at the site of the dural tear [5]. All of our patients were managed conservatively through bed rest, in prone position, with acetazolamide (carbonic anhydrase inhibitor) and antibiotics. All the study patients recovered within 1 week of treatment.

Our study generated data regarding postoperative CSF leakage in local patients undergoing a laminectomy that 
would facilitate proper and careful tissue handling by surgeons. We believe that our study will help in counseling patients and their attendants on the expected morbidity (associated with CSF leakage) and the resulting prolonged hospital stay. This will help in decision making and setting expectations. However, there are certain limitations to our study. Firstly, the study was based on a relatively small number of patients admitted to a particular urban hospital. Secondly, we did not assess the severity of potential covariates. For instance, hypertension, diabetes, smoking and drug use were recorded simply as 'yes' or 'no'. Details of these potential covariates such as smoking rate or duration of smoking, average glucose or hemoglobin $\mathrm{A} 1 \mathrm{C}$ for diabetes and drug dosage for drug abuse were not inquired. Hence, we recommend additional studies involving larger sample sizes with nationwide data to fully understand the risk factors associated with incidental durotomies.

\section{Conclusions}

This study indicated that smoking and diabetes are significant predictors of incidental durotomy. Hence cessation of smoking and good glycemic control among regional patients well before surgery can be of utmost importance in preventing dural tear. However, larger clinical studies should be conducted to understand the predisposing factors associated with this serious complication for better planning of spinal surgeries in the future.

\section{Conflict of Interest}

No potential conflict of interest relevant to this article was reported.

\section{References}

1. Kalff R, Ewald C, Waschke A, Gobisch L, Hopf C. Degenerative lumbar spinal stenosis in older people: current treatment options. Dtsch Arztebl Int 2013;
110:613-23.

2. Joaquim AF, Sansur CA, Hamilton DK, Shaffrey CI. Degenerative lumbar stenosis: update. Arq Neuropsiquiatr 2009;67:553-8.

3. ECRI Health Technology Assessment Group. Treatment of degenerative lumbar spinal stenosis. Evid Rep Technol Assess (Summ) 2001;(32):1-5.

4. Gunzburg R, Szpalski M. The conservative surgical treatment of lumbar spinal stenosis in the elderly. Eur Spine J 2003;12 Suppl 2:S176-80.

5. Kalevski SK, Peev NA, Haritonov DG. Incidental Dural Tears in lumbar decompressive surgery: incidence, causes, treatment, results. Asian J Neurosurg 2010;5:54-9.

6. Guerin P, El Fegoun AB, Obeid I, et al. Incidental durotomy during spine surgery: incidence, management and complications. A retrospective review. Injury 2012;43:397-401.

7. Williams BJ, Sansur CA, Smith JS, et al. Incidence of unintended durotomy in spine surgery based on 108,478 cases. Neurosurgery 2011;68:117-23.

8. Baker GA, Cizik AM, Bransford RJ, et al. Risk factors for unintended durotomy during spine surgery: a multivariate analysis. Spine J 2012;12:121-6.

9. Desai A, Ball PA, Bekelis K, et al. SPORT: does incidental durotomy affect long-term outcomes in cases of spinal stenosis? Neurosurgery 2011;69:38-44.

10. Yoshihara H, Yoneoka D. Incidental dural tear in lumbar spinal decompression and discectomy: analysis of a nationwide database. Arch Orthop Trauma Surg 2013;133:1501-8.

11. Silverstein P. Smoking and wound healing. Am J Med 1992;93:22S-24S.

12. Rosenberg CS. Wound healing in the patient with diabetes mellitus. Nurs Clin North Am 1990;25:24761.

13. Ahn N, Ahn U, Post Z, et al. Smoking, diabetes and excessive preoperative epidural steroid administration are risk factors for intraoperative dural tears. Spine J 2004;4:S22. 